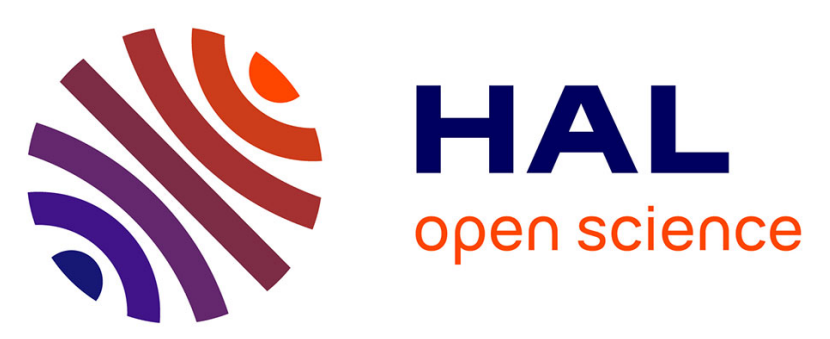

\title{
Analyse de l'activité question-réponse d'une pharmacie à usage intérieur. Exemple de la gestion des interactions médicamenteuses
}

\author{
Elodie Jacqueroux, Laure Lalande, Nadia Meddour, Charlotte Papailhau, \\ Elodie Bernard, Charlotte Charroin, Juliette Perichou, Bruno Charpiat, \\ François Locher, Stephan Garcia
}

\section{To cite this version:}

Elodie Jacqueroux, Laure Lalande, Nadia Meddour, Charlotte Papailhau, Elodie Bernard, et al.. Analyse de l'activité question-réponse d'une pharmacie à usage intérieur. Exemple de la gestion des interactions médicamenteuses. Annales Pharmaceutiques Française, 2014, 10.1016/j.pharma.2014.10.004. 10.1016/j.pharma.2014.10.004 . hal-01084158

\section{HAL Id: hal-01084158 https://hcl.hal.science/hal-01084158}

Submitted on 18 Nov 2014

HAL is a multi-disciplinary open access archive for the deposit and dissemination of scientific research documents, whether they are published or not. The documents may come from teaching and research institutions in France or abroad, or from public or private research centers.
L'archive ouverte pluridisciplinaire HAL, est destinée au dépôt et à la diffusion de documents scientifiques de niveau recherche, publiés ou non, émanant des établissements d'enseignement et de recherche français ou étrangers, des laboratoires publics ou privés. 
Analyse de l'activité question-réponse d'une pharmacie à usage intérieur. Exemple de la gestion des interactions médicamenteuses

Analysis of the question-answer activity of a hospital pharmacy. Example of the handling of drug-interactions

Jacqueroux Elodie ${ }^{1}$, Lalande Laure ${ }^{2}$, Meddour Nadia ${ }^{2}$, Papailhau Charlotte ${ }^{1}$, Bernard Elodie $^{2}$, Charroin Charlotte ${ }^{2}$, Perichou Juliette ${ }^{2}$, Charpiat Bruno $^{2}$, Locher François ${ }^{1}$, Garcia Stephan ${ }^{1}$

1. Centre de documentation et d'information pharmaceutiques, pharmacie centrale, hospices civils de Lyon, 69561 Saint-Genis Laval cedex, France

2. Service pharmacie, hôpital de la Croix-Rousse, hospices civils de Lyon, 69317 Lyon cedex 04, France

Auteur correspondant : Stephan Garcia

Tel : 04788633 53/ Fax : 0472678876 / mail : stephan.garcia@chu-lyon.fr 


\section{Analyse de l'activité question-réponse d'une pharmacie à usage intérieur. Exemple de la gestion des interactions médicamenteuses

\begin{abstract}
Analysis of the question-answer activity of a hospital pharmacy. Example of the
\end{abstract} handling of drug-interactions}

\section{Résumé}

\section{Introduction :}

La prise en charge médicamenteuse d'un patient génère des questions chez les professionnels de santé impliqués. Certaines vont nécessiter une recherche avancée. Le pharmacien hospitalier est situé à l'interface entre les prescripteurs, le personnel soignant et le médicament et est sollicité pour répondre à ces demandes. Des études menées à l'étranger, ont montré que cette activité question-réponse représente une part significative du travail quotidien. En France, ce sujet est peu exploré. L'objectif de notre travail a été de connaître cette activité : volume et nature des questions, situations cliniques, temps consacré, médicaments concernés, sources d'information utilisées.

\section{Matériels et méthodes :}

Une étude prospective a été menée au sein de la pharmacie d'un centre hospitalouniversitaire. Toutes les demandes traitées par l'équipe pharmaceutique, ayant nécessité un travail de recherche, d'analyse et de rédaction d'une réponse ont été recensées.

\section{Résultats :}

Cent-une questions ont été analysées, émanant pour plus des deux tiers de médecins ou d'internes en médecine. Près de la moitié concernait des analyses d'interactions médicamenteuses et presqu'un quart d'entre elles n'était pas mentionné dans le Résumé des Caractéristiques des spécialités concernées. Une conduite à tenir a été proposée dans $91,5 \%$ des cas. Le temps consacré à la recherche d'information variait de moins de 30 minutes à plus de 8 heures. 


\section{Discussion et conclusion :}

Cette étude illustre l'activité question-réponse d'une Pharmacie à Usage Intérieur, actuellement absente des indicateurs d'activité pharmaceutique. Une grande partie porte sur l'analyse et la gestion des interactions médicamenteuses et nécessite un temps de travail conséquent.

\section{Introduction :}

The medical care of patients generates questions among healthcare professionals. Some will necessitate an advanced research. The hospital pharmacist is at the interface between prescribers, caregivers and the medicines and is requested to answer these requests. Studies conducted in others countries showed that this question-answer activity represents a significant amount of time in daily work. In France, this topic was poorly explored. The objective of our work was to study the volume and the type of questions, the clinical situations, the time required, the medicines implicated and the sources of information used.

\section{Materials and methods :}

A prospective study was conducted in the pharmacy of a university hospital. All the requests answered by the pharmaceutical team, which needed a specific research, analysis and writing of an answer were collected.

\section{Results :}

A hundred and one questions were analyzed, originating from doctors or medicals interns. Almost half concerned drug interactions, and among them, almost a fourth were not mentioned in the Summary of Product Characteristics of the medicines involved. A pharmaceutical advice was provided in $91,5 \%$ of the cases. Time dedicated to the research varied between less than 30 minutes and more than 8 hours.

\section{Discussion and conclusion :}

This study illustrates the question-answer activity of a hospital pharmacy, which is currently not taken into account as an indicator of pharmaceutical activity. A large part concerns 
analysis and management of drug interactions and requires a significant amount of pharmaceutical time.

Mots-clés : Information pharmaceutique, Interventions pharmaceutiques, Interactions médicamenteuses, Hôpital, Pharmacie clinique.

Key-words : Pharmaceutical information, Pharmaceutical interventions, Drug interactions, Hospital, Clinical pharmacy.

\section{Introduction :}

Lors de la prise en charge médicale d'un patient, médecins et pharmaciens sont amenés à se questionner sur la thérapeutique médicamenteuse. Le nombre et la nature des questions varient selon le contexte clinique notamment [1-3]. D'après une revue récente de la littérature, plus de $30 \%$ des interrogations de cliniciens portent sur le traitement médicamenteux de leurs patients [3]. Le personnel soignant peut aussi être confronté à des interrogations lors de la préparation et/ou l'administration des médicaments. Une partie de ces demandes trouve une réponse grâce à la consultation de bases de données sur le médicament [1]. D’autres, plus complexes, ou pour lesquelles les bases de données les plus courantes ne sont pas renseignées, vont nécessiter une recherche plus importante. Le manque de temps est régulièrement mis en avant par les professionnels de santé comme un obstacle pour trouver eux-mêmes la réponse à ces questions [1, 3].

Le pharmacien hospitalier travaille en collaboration avec les prescripteurs et le personnel soignant. II dispose d'une expertise sur le médicament. Cela en fait un interlocuteur privilégié lorsqu'une question émerge concernant la prise en charge médicamenteuse d'un patient (PCMP). Un travail mené aux Etats-Unis, a montré qu'il est sollicité plusieurs fois par jour par d'autres professionnels de santé [4].

L'activité question-réponse des pharmaciens dans les hôpitaux étrangers a fait l'objet d'études qui ont montré qu'elle représente une part significative du travail quotidien [5, 6]. En 
France ce sujet reste peu exploré [7, 8]. Une étude a été menée en 1999-2000 pour évaluer les questions posée au personnel d'une pharmacie à usage intérieur (PUI), quelle que soit leur nature. Les auteurs ont utilisé un logiciel dédié pour les saisir et les archiver. Cependant, ils n'ont pas analysé précisément le thème des questions, les situations cliniques ou encore le statut des demandeurs. Certains établissements ont également rapporté disposer d'un service d'information dédié, mais les publications sont pour la plupart anciennes [9-13]. Ainsi, il nous a semblé intéressant d'étudier spécifiquement les requêtes émanant des médecins et du personnel soignant à destination des pharmaciens hospitaliers, que ce soit à la PUI ou dans les services de soin.

L'objectif de notre travail a été de connaître cette activité en s'intéressant au volume et à la nature des questions, aux situations cliniques, au temps consacré, aux médicaments concernés ainsi qu'aux sources d'information utilisées pour répondre.

\section{Matériels et méthodes:}

Une étude prospective a été menée entre le 10 mai 2012 et le 5 février 2014 . Toutes les questions traitées par l'équipe pharmaceutique de la PUI d'un groupement hospitalier, ayant nécessité un travail de recherche, d'analyse et de rédaction d'une réponse transmise sous forme d'un courrier, ont été recensées. Toutes celles dont la réponse pouvait être fournie par simple consultation du Résumé des Caractéristiques du Produit (RCP) ont été exclues. Ces questions ont été adressées directement à la PUI ou recueillies par l'équipe pharmaceutique dans les services de soins. Les questions ont été saisies dans une base de données nommée GULPER, intégrée dans le système d'information hospitalier des Hospices Civils de Lyon (HCL), Cristalnet®. Les informations dans la base de données étaient les suivantes : nom et fonction du demandeur, unité de soins, situation clinique et références documentaires utilisées. Chaque question a ensuite été relue et indexée avec des mots-clés par l'interne du Centre de Documentation et d'Information Pharmaceutiques (CDIP). Pour cela, un thésaurus de termes pharmaceutiques a été rédigé, en s'appuyant sur des expériences similaires de 
construction d'une base de données question-réponse [14, 15]. L'extraction et l'analyse des données ont été réalisées par l'équipe du CDIP à l'aide du logiciel Excel®.

\section{Résultats:}

Au cours de la période d'étude, 101 questions ont été enregistrées et analysées. Le statut du demandeur était précisé dans 99 cas. Dans 42,4 \% il s'agissait d'un médecin, dans 26,3\% d'un interne en médecine, dans $18,2 \%$ de pharmaciens en situation de soin dans un service médical, dans $9,1 \%$ de personnels soignants et dans $4 \%$ d'autres personnes (une patiente, une diététicienne, un externe, un cadre de santé). Le service de soins dont émanait la demande était précisé dans $95 \%$ de cas $(n=96)$. Dix-huit questions provenaient du service des maladies infectieuses, 11 du service d'addictologie, 9 de pneumologie, 8 d'hépatogastrologie, 7 de chirurgie, 6 du centre de dépistage anonyme et gratuit, 6 des urgences, 5 de gériatrie, et 5 de la pharmacie. Le reste des questions a été reçu à part égale des services de gynécologie, réanimation, cardiologie, néonatologie, obstétrique, nutrition, médecine interne et hygiène.

La quasi-totalité des questions portait sur le médicament $(n=96)$. Les autres concernant des produits d'aromathérapie, de phytothérapie ou de nutrition. Les thèmes des questions sont résumés dans le tableau 1 . Une fois sur deux $(n=47)$, cela concernait une recherche d'interactions médicamenteuses (IAM). Le tableau 2 présente les principales classes pharmacothérapeutiques concernées. II s'agissait majoritairement de médicaments antiinfectieux et du système nerveux central.

Les questions portant sur les IAM ont été analysées plus précisément : elles se sont avérées refléter deux situations distinctes, qui sont présentées et illustrées dans le tableau 3.

Parmi les 47 recherches d'IAM, une ou plusieurs ont été documentées dans 39 cas (83\%). Elles étaient mentionnées dans le RCP ou dans le thésaurus des IAM de l'ANSM dans 64\% ; rapportées par d'autres bases de données ou dans la littérature sous forme de données primaires dans $25,7 \%$ et aucune donnée n'était disponible pour permettre de proposer une 
conduite à tenir dans 10,2\%. L'absence d'IAM a été validée par le pharmacien dans 17\% $(n=8)$. Au total, un avis pharmaceutique a donc été proposé 9 fois sur $10(n=43)$, qu'il s'agisse d'une alternative thérapeutique, d'une adaptation de posologie, d'une modification du plan de prise ou de la mise en place d'un suivi thérapeutique.

Parmi les demandes hors IAM, aucune information n'a été retrouvée pour une seule question. Elle concernait la possibilité d'administrer la céfoxitine par voie sous cutanée. Le pharmacien a donc expliqué que cette voie d'administration ne pouvait pas être envisagée.

Le temps consacré à la recherche d'information était renseigné dans 62 cas sur 101 (61,3\%). II était inférieur à 30 minutes dans $29 \%$, compris entre 30 minutes et 1 heure dans $30,6 \%$, entre 1 heure et 4 heures dans $32,2 \%$, entre 4 heures et 8 heures dans $4,8 \%$ et supérieur à 8 heures dans 3,2\%.

Pour chaque question, la valeur médiane du nombre de document consultés et référencés a été de 3 [valeurs extrêmes: 1-32]. Plusieurs types de ressources documentaires ont été identifiés :

- des documents publiés ou validés par des organismes publics de santé, tels que l'ANSM, la HAS ou les Omedit, dans $57,8 \%$ des cas ;

- des bases de données en accès libre et gratuit pour les professionnels de santé rédigées par des professionnels de santé et/ou universitaires, à partir de sources clairement identifiées, dans $47 \%$ des cas. II s'agit par exemple de la base Stabilis ${ }^{\circledR}$, du site de la Pharmacie des Hôpitaux Universitaires de Genève, de l'Association pour le Développement de l'Internet en Pharmacie, ou encore du site Guide de prescription et rein. On peut aussi mentionner les sites universitaires spécialisés sur les interactions avec les cytochromes (http://www.pkis.org/), les médicaments torsadogènes (www. http://crediblemeds.org/) ou les médicaments du VIH et des hépatites (www.hiv-druginteractions.org ; www.hivclinic.ca) ; 
- des articles scientifiques obtenus après interrogation de base de données bibliographiques comme Pubmed, dans $47 \%$ des cas. Le nombre de références de données primaires varie de 1 à 8 , mais dans $68 \%$ il est inférieur ou égal à 2 articles. On peut noter que les revues citées appartiennent au champ pharmaceutique mais aussi médical, en lien avec la ou les indication(s) traitée(s) (allergologie, dermatologie, oncologie,...). Ces revues sont très diverses et aucune revue scientifique n’a été utilisée préférentiellement ;

- des documents émanant des firmes pharmaceutiques dans $21 \%$ des situations ;

- des sites ou ouvrages de références étrangers, d'accès payant dans $20 \%$ des cas, tels que le « Martindale », ou l'ouvrage « Handbook on injectable drugs »;

- le centre régional de pharmacovigilance ou un confrère pharmacien dans $5 \%$

- des sites internet généralistes ou grand public, dans $4 \%$ des situations (par exemple pour identifier la composition d'un complément alimentaire).

\section{Discussion :}

Ce travail nous a permis de connaître le volume et la nature des questions reçues par une PUI, le temps consacré, ainsi que les sources d'information utilisées pour répondre. Un travail préliminaire nous avait montré que les PUI des HCL, sont quotidiennement sollicitées pour des questions en lien avec la prise en charge médicale d'un patient (données non publiées). Cependant, les pratiques se sont avérées très hétérogènes, en termes de saisie et d'archivage des questions et des réponses transmises. D'une manière générale, l'activité question-réponse est absente des indicateurs d'activité pharmaceutique.

A notre connaissance, il s'agit de la première étude française traitant de cette question de manière détaillée. Un précédent travail a porté sur les questions posées au personnel d'une PUI, mais l'analyse était plus succincte et ne permettait pas distinguer les questions essentiellement pratiques, par exemple la disponibilité commerciale d'un médicament, de demandes plus complexes [8]. Un travail plus ancien a aussi étudié les appels téléphoniques 
reçus par une PUI et concernant une demande d'information sur le médicament, mais les résultats n'ont pas été détaillés [7]. Des études menées à l'étranger ont montré que ces demandes peuvent être à l'origine d'une grande partie des interventions pharmaceutiques ou des avis pharmaceutiques émis [5, 6].

Nos résultats mettent en lumière que les demandes émanent de tous les personnels médicaux et soignants, même si les principaux demandeurs sont des médecins. Cela peut s'expliquer par le type de questions que nous avons analysées, plus complexes et qui proviennent plutôt des prescripteurs que des personnels soignants. La majorité des patients concernés sont atteints d'infections par le VIH, le VHC ou le VHB, insuffisants et/ou transplantés hépatiques, atteints de comorbidités telles qu'infections virales ou tuberculose associées à d'importants troubles psychiatriques. La prise en charge médicamenteuse simultanée de ces pathologies aboutit à des situations complexes. De surcroit, d'après le tableau 2 près de la moitié des médicaments concernés sont à marge thérapeutique étroite et à risque d'IAM.

Les demandes portent sur différents aspects de la PCMP, de la prescription jusqu'à l'administration, mais près de $50 \%$ concerne la recherche d'IAM avant prescription. Cela est probablement lié au statut des demandeurs, principalement des médecins. Une enquête menée aux Etats-Unis auprès des internes en médecine, avait montré que les IAM étaient le troisième thème pour lequel ils sollicitaient les pharmaciens hospitaliers, derrière les questions de posologie et toxicité [16]. Près d'un tiers des questions porte à parts égales, sur les modalités d'administration et sur les propriétés physico-chimiques, pharmacocinétiques ou pharmacodynamiques. Les informations fournies par les RCP sont fréquemment insuffisantes pour répondre à un usage hors $A M M$, mais validé en pratique clinique (administration sous cutanée, médicaments perfusés simultanément, comprimés écrasés). En ce qui concerne les IAM, dans près de deux tiers des cas, le prescripteur demande au pharmacien de lui proposer directement un médicament, soit entre plusieurs molécules d'une 
même classe, soit sur la base de l'indication souhaitée (exemple: «traitement anxiolytique ») et des traitements concomitants. Dans ces situations, le pharmacien a un rôle d'aide à la prescription, qui va au-delà de l'analyse a posteriori des prescriptions lors de la dispensation.

Les IAM augmentent la morbidité et la mortalité et sont responsables d'hospitalisations [17 21]. En France, les études concernant les IAM restent rares [22]. Certaines se rapportent à des patients traités en ambulatoire [23, 24], d'autres à des ordonnances de ville qui ont été analysées par des médecins ou des pharmaciens à la suite d'une hospitalisation des patients [17, 25-27]. La recherche et le signalement des IAM constituent une part significative du travail du pharmacien lors de l'analyse et la validation des prescriptions. Quelques équipes ayant développé l'analyse de prescriptions au sein de leurs hôpitaux respectifs rapportent des fréquences d'interventions concernant les interactions médicamenteuses qui varient de $5 \%$ à $30 \%$ [28-33]. Néanmoins, les équipes qui ont développé ce type d'activités sont encore peu nombreuses et ces travaux ne donnent pas ou peu de détails sur la nature des interactions les plus fréquemment rencontrées (médicaments et mécanismes impliqués). Les sources documentaires employées pour argumenter les interventions pharmaceutiques ne sont généralement pas citées. Les conditions d'interventions et le mode de communication sont peu détaillés [22]. Un travail multicentrique a montré une grande variabilité quant à la nature et la fréquence des IAM détectées et signalées par les établissements participants [34]. Cependant, les variables qui pourraient certainement expliquer ces différences, sont les connaissances du praticien, la nature des outils à sa disposition, les conditions d'usage de ces derniers et le mode de communication avec les cliniciens [34, 35].

La rédaction de la prescription par un dialogue conjoint entre le prescripteur et le pharmacien, en amont de la dispensation, permet de réduire le risque de survenue d'une IAM. Notre étude montre que le pharmacien a documenté des interactions non répertoriées 
dans le thésaurus de l'ANSM (et donc non documentées par les logiciels d'aide à la prescription), en consultant d'autres sources d'informations et des données primaires. II a ainsi pu proposer une conduite à tenir argumentée.

Une étude multicentrique française, nous a appris que lors de la détection d'IAM pendant l'analyse de prescription, les pharmaciens proposaient le plus souvent un suivi thérapeutique, illustrant ainsi la difficulté d'évaluer précisément la situation clinique et l'impact potentiel de l'IAM [36]. Outre une meilleure gestion des IAM, ce dialogue permet au pharmacien d'obtenir plus d'informations cliniques et de renforcer la pertinence des avis pharmaceutiques, par rapport à une simple intervention informatique sur une prescription déjà rédigée [30]. Cela peut aussi concourir à leur meilleure acceptation.

Notre travail apporte également un éclairage sur le temps de travail requis pour étudier une question, entreprendre les recherches documentaires nécessaires, analyser le contenu des documents puis rédiger une réponse.

Dans plus de $90 \%$ des cas, le temps de recherche a été inférieur à une demi-journée. Pour le reste le temps requis apparaît supérieur à 4 voire 8 heures. Cette information est probablement inconnue des décideurs hospitaliers. La médiane des sources d'information est de trois et pour un nombre important de questions, les réponses s'appuyaient sur un socle commun d'outils documentaires. Ainsi, la liste des sources est relativement réduite et limitée principalement à des bases de données de référence fréquemment accessibles en ligne, institutionnelles ou non mais avec des modalités de rédaction, validation et mise à jour clairement définies ou encore directement à la littérature scientifique validée par les pairs. Dans un environnement documentaire pharmaceutique de plus en plus vaste, le choix et la bonne utilisation des sources sont primordiaux pour répondre pertinemment à une question [37]. On voit aussi que les firmes pharmaceutiques restent une source régulièrement consultée. Une enquête menée auprès de pharmaciens hospitaliers français, a aussi montré qu'ils utilisent principalement des bases électroniques d'information pharmaceutique, les 
sites des agences de santé et des sites spécialisés. Cependant, les collègues pharmaciens étaient cités comme source d'information dans $58 \%$ des cas [9].

Cette étude présente plusieurs limites : tout d'abord la nature des questions analysées. Elle est le reflet des spécialités médicales de l'établissement. En effet, les thèmes ainsi que le temps de recherche et d'analyse ne seraient pas forcément transposables dans d'autres types d'établissements tels que psychiatriques ou de lutte contre le cancer. D'autres études de même nature doivent être entreprises par des confrères dans des disciplines médicales différentes.

Malgré une formation des internes de la PUI à l'utilisation de l'outil informatique, la rédaction d'une procédure et un suivi régulier par les internes du CDIP, de nombreuses fiches étaient incomplètes à l'issue de la période d'étude, ce qui a limité l'analyse. De même, il est probable que le recueil des questions traitées, qui s'est souvent fait a posteriori, ne soit pas exhaustif.

Le nombre de questions rapporté peut paraître peu élevé, notamment au regard des sollicitations quotidiennes dont fait l'objet une PUI. En 2000, Vanderverren et al ont rapporté le chiffre de près de 20 questions par mois environ, mais $48 \%$ portaient sur la disponibilité commerciale, la substitution ou une demande d'information sur un nouveau médicament [8]. Un travail préliminaire, mené pour recenser toutes les questions reçues à la PUI avait rapporté des chiffre similaires en termes de volume (données non publiées) Pour le travail décrit ici, nous avons choisi de nous limiter aux questions portant sur la PCMP et ayant nécessité une recherche documentaire et la rédaction d'un avis argumenté. Cependant, cette définition reste subjective et des demandes auxquelles les pharmaciens ou internes ont pu répondre rapidement, par exemple sur la base de leur expérience ou en consultant le $\mathrm{RCP}$, n'ont pas été saisies, même si elles pouvaient avoir un impact sur la PCMP et sur le temps de travail pharmaceutique. Une autre raison peut expliquer ce chiffre: lorsque le médecin ou le personnel soignant est confronté à une problématique, il mène d'abord une 
recherche auprès de ses collègues de travail ou sur des bases de données auxquelles il a accès. La PUI ne semble sollicitée qu'en deuxième ligne. Des enquêtes menées auprès de médecins hospitaliers, montrent qu'ils se tournent préférentiellement vers des bases de données électroniques ou des ouvrages de référence par rapport à un autre professionnel de santé $[3,38]$. II est aussi possible que les demandeurs se tournent plus spontanément vers l'équipe pharmaceutique si elle dispose d'un service question-réponse formalisé, clairement identifié et aisément joignable. Plusieurs études ont montré l'impact positif que ce genre de structures peut avoir sur la PCMP [39]. De même, un pharmacien ou un interne présent dans un service de soins sera plus fréquemment sollicité. L'acceptabilité par le prescripteur n'a pas été mesurée et l'impact de l'avis pharmaceutique sur la prise en charge du patient n'a pas été étudié. Cependant, on peut penser que le demandeur est d'autant plus à même de prendre en compte un avis, qu'il l'a lui-même sollicité et qu'il lui est nécessaire pour poursuivre la prise en charge de son patient. II conviendrait cependant d'interroger les demandeurs sur leur niveau de satisfaction et l'utilisation qu'ils ont fait de la réponse apportée [39].

Plusieurs perspectives se dégagent de ce travail. Nous avons utilisé un outil informatique pour saisir, indexer et analyser les demandes. A l'avenir, il peut être intéressant de développer une base question-réponse, accessible à tous les pharmaciens. Ce partage pourrait constituer une aide aux confrères rencontrant des situations cliniques similaires à celles déjà abordées. Ceci ouvrirait aussi la porte à une analyse critique des réponses déjà fournies. Une telle base pourrait aussi être utilisée comme indicateur de cette activité [8].

\section{Conclusion :}

Ce travail illustre un volet encore peu connu de l'activité d'une PUI. II montre de quelle manière elle est sollicitée par les autres professionnels de santé sur des questions en lien avec la PCMP. Une grande partie porte sur la gestion des IAM et les réponses apportées permettent d'anticiper des situations cliniques potentiellement compliquées. Le temps de 
travail apparaît conséquent. De même que de nombreux confrères codent et enregistrent le nombre et la nature des interventions pharmaceutiques faites au cours de l'analyse des prescriptions, nous les invitons à enregistrer cette activité question-réponse afin d'une part, de la rendre lisible auprès des prescripteurs et des décideurs et d'autre part, d'objectiver la plus-value pharmaceutique qui y est associée.

\section{Déclaration d'intérêts}

Les auteurs déclarent ne pas avoir de conflits d'intérêts en relation avec cet article.

\section{Remerciements}

Les auteurs remercient M. Boucquin pour l'aide à la recherche bibliographique.

\section{Références:}

1. Davies K, Harrison J. The information-seeking behaviour of doctors: a review of the evidence. Health Inf Libr J. 2007;24(2):78-94.

2. Davies K. Quantifying the information needs of doctors in the UK using clinical librarians. Health Inf Libr J. 2009;26(4):289-97.

3. Del Fiol G, Workman TE, Gorman PN. Clinical Questions Raised by Clinicians at the Point of Care: A Systematic Review. JAMA Intern Med. 2014;174(5):710-8.

4. Schweigert BF, Oppenheimer PR, Smith WE. Hospital pharmacists as a source of drug information for physicians and nurses. Am J Hosp Pharm. 1982;39(1):74-7.

5. Langebrake $\mathrm{C}$, Hilgarth $\mathrm{H}$. Clinical pharmacists' interventions in a German university hospital. Pharm World Sci. 2010;32(2):194-9.

6. Lada P, Delgado G Jr. Documentation of pharmacists' interventions in an emergency department and associated cost avoidance. Am J Health Syst Pharm. 2007;64(1):63-8.

7. Perrin A, Perrin P, Hoffmann M, Hoffmann M. Besoins en information pharmaceutique à l'hôpital. Rev Fr Santé Publ. 1985;32:51-5.

8. Vanderverren I, Tolla C, Talon D, Sinegre M. Intérêt d'un logiciel questions-réponses pharmaceutiques dans une pharmacie à usage intérieur. Act Pharm Biol Clin. 2000 11:241-5. 
9. Hubault M, Locher F, Garcia S. Enquête auprès de pharmaciens hospitaliers sur l'information pharmaceutique : quels besoins ? Quelles sources ? Quelle utilisation et quel impact d'un centre d'information pharmaceutique ? Pharm Hosp Clin. 2014;49(1):4-10.

10. Belghazi L, Joos A, N'koudmongo C, Locher F, Garcia S. Analyse de l'activité "Question - Réponse » du Centre de documentation et d'information pharmaceutiques des hospices civils de Lyon et enquête auprès des pharmaciens hospitaliers utilisateurs. Pharm Hosp Clin. 2012;47(2):83-90.

11. Bador $P$, Derharoutunian $C$, Locher $F$, Revol C. information et documentation pharmaceutiques en région Rhône-Alpes. Lyon Pharm. 1992;43(1):51-6.

12. Müllerová $H$, Vlcek J. European drug information centres--survey of activities. Pharm World Sci. 1998;20(3):131-5.

13. Taggiasco N, Sarrut B, Doreau CG. European survey of independent drug information centers. Ann Pharmacother. 1992;26(3):422-8.

14. Dugas M, Weinzierl S, Pecar A, Hasford J. An intranet database for a university hospital drug information center. Am J Health Syst Pharm. 2001;58(9):799-802.

15. Ohman B, Lyrvall H, Alván G. Use of DRUGLINE--a question-and-answer database. Ann Pharmacother. 1993;27(3):278-84.

16. Lantos RL. What questions do resident physicians ask pharmacists? Pharm Times. 1984;50(9):28-9.

17. Doucet J, Chassagne P, Trivalle C, Landrin I, Pauty MD, Kadri N, et al. Drug-drug interactions related to hospital admissions in older adults: a prospective study of 1000 patients. J Am Geriatr Soc. 1996;44(8):944-8.

18. Hamilton RA, Briceland LL, Andritz MH. Frequency of hospitalization after exposure to known drug-drug interactions in a Medicaid population. Pharmacotherapy. 1998;18(5):1112-20.

19. McDonnell PJ, Jacobs MR. Hospital admissions resulting from preventable adverse drug reactions. Ann Pharmacother. 2002;36(9):1331-6.

20. Juurlink DN, Mamdani M, Kopp A, Laupacis A, Redelmeier DA. Drug-drug interactions among elderly patients hospitalized for drug toxicity. JAMA. 2003;289(13):1652-8.

21. Becker ML, Kallewaard M, Caspers PWJ, Visser LE, Leufkens HGM, Stricker BHC. Hospitalisations and emergency department visits due to drug-drug interactions: a literature review. Pharmacoepidemiol Drug Saf. 2007;16(6):641-51.

22. Charpiat B, Allenet B, Roubille R, Escofier L, Bedouch $P$, Juste $M$, et al. Facteurs à prendre en considération pour la gestion des interactions médicamenteuses en pratique clinique. Presse Médicale. 2008;37(4, Part 2):654-64. 
23. Rémy N, Lapeyre-Mestre M, Bareille MP, Bagheri H, Montastruc JL. Interactions médicamenteuses: à propos d'une étude prospective pilote en médecine générale libérale. Thérapie. 2000;55(3):395-8.

24. Guédon-Moreau L, Ducrocq D, Duc M-F, Quieureux Y, L'Hôte C, Deligne J, et al. Absolute contraindications in relation to potential drug interactions in outpatient prescriptions: analysis of the first five million prescriptions in 1999. Eur J Clin Pharmacol. 2004;59(12):899-904.

25. Manchon ND, Bercoff E, Lemarchand P, Chassagne P, Senant J, Bourreille J. Fréquence et gravité des interactions médicamenteuses dans une population âgée: étude prospective concernant 639 malades. Rev Médecine Interne. 1989;10(6):521-5.

26. Barré E, Bisseux L, Chiadmi F, Toledano A, Cisternino S, Schlatter J, et al. Interactions médicamenteuses dans une population âgée : Étude prospective de leur fréquence et de leur gravité auprès de 56 malades. Presse Médicale. 2005;34(12):837-41.

27. Doucet J, Kalimoutou S, Chédru C, Remy E, Cabrejo L, Méliot C, et al. Les prescriptions de médicaments anticholinestérasiques sont-elles adaptées au contexte médical au cours de la démence de type Alzheimer ?. Thérapie. 2006;61(1):23-7.

28. Zamparutti $P$, Le Duff $M$. Evaluation de la fréquence et de la nature des interventions pharmaceutiques effectuées depuis la pharmacie de l'hôpital comparativement à celles effectuées dans une unité de soins. Pharm Hosp. 1995;30(120):31-3.

29. Vernardet S, Bossaert S, Livrozet A, Pont E, Charpiat B. Validation pharmaceutique des prescriptions hospitalières: Intervention et analyse sur cinq ans. Presse Médicale. 2005;34(14):990-6.

30. Bedouch P, Allenet B, Labarere J, Brudieu E, Chen C, Chevrot D, et al. Diffusion des opinions pharmaceutiques dans le cadre d'une activité de pharmacie clinique en unité de soins. Thérapie. 2005;60(5):515-22.

31. Gaillard K, Bohand X, Beranger C, Boulliat C, Guevel C. Évaluation des interventions pharmaceutiques à l'hôpital d'instruction des armées Sainte-Anne dans le cadre d'une dispensation journalière individuelle et nominative. J Pharm Clin. 2006;(25):39-47.

32. Garreau I, Derharoutunian C, Roubille R, Galtier H. Assessment of pharmacy interventions after drug orders review: a 34 months retrospective study (july 94 - april 97). Process in clinical pharmacy, future therapies and clinical pharmacy. Tours; 1997.

33. Demange C. Analyse pharmaceutique des prescriptions en unité de soins à l'aide de la fiche d'intervention de la Société française de pharmacie clinique. J Pharm Clin. 2007;26(1):45-52.

34. Roubille R, Charpiat B, Escofier L, Bedouch P, Juste M, Rose F-X. Potentiel d'étude de la base ACT-IP $\square$ : illustration avec les interactions médicamenteuses. J Pharm Clin. 2007;26(4):261-6.

35. Dumortier G, VanNieuwenhuyse V, Zerrouk A, Chebili S, Glikman J, Degrassat K. Démarche qualité dans le circuit médicament grâce à l'analyse pharmaceutique des 
ordonnances dans un établissement de soins psychiatriques. Encéphale.

1999;(25):323-8.

36. Bedouch P, Charpiat B, Conort O, Rose F-X, Escofier L, Juste M, et al. Assessment of clinical pharmacists' interventions in French hospitals: results of a multicenter study.

Ann Pharmacother. 2008;42(7):1095-103.

37. Jean-Bart E, Charpiat B, Bourdon O, Grassin J, Bornet C, Dode X, et al. État des lieux des outils documentaires pour l'analyse d'ordonnance accessibles sur Internet : pouvons-nous conjuguer multiplicité avec efficacité ? Pharm Hosp Clin. 2013;48(2):95-102.

38. Graber MA, Randles BD, Ely JW, Monnahan J. Answering clinical questions in the ED. Am J Emerg Med. 2008;26(2):144-7.

39. Bramley DM, Innes AJ, Duggan C, Oborne CA. The impact of Medicines Information enquiry answering on patient care and outcomes. Int J Pharm Pract.

2013;21(6):393-404. 
Tableau 1. Thèmes des questions reçues par l'équipe pharmaceutique.

Table 1. Categories of questions received by the pharmaceutical team

\begin{tabular}{|c|c|}
\hline Thèmes des questions & Pourcentage $^{\star}$ \\
\hline Interactions médicamenteuses & $46,5 \%$ \\
\hline Modalités d'administration & $17,8 \%$ \\
- Voie injectable & $10 \%$ \\
- Voie orale & $4,9 \%$ \\
\hline - Autres voies & $2,9 \%$ \\
\hline Propriétés physico-chimiques, \\
pharmacocinétiques ou \\
pharmacodynamiques \\
Effets indésirables - Toxicité \\
Bouverture/reconstitution)
\end{tabular}

*Le total est supérieur à $100 \%$ car une même question pouvait aborder plusieurs thèmes. 
Tableau 2. Principales classes pharmacothérapeutiques des médicaments concernés par les demandes traitées par l'équipe pharmaceutique.

Table 2. Main pharmacotherapeutic groups of medicines involved in the questions treated by the pharmaceutical team

$\left.\begin{array}{|c|cc|}\hline \text { Classe pharmacothérapeutique } & \text { Pourcentage } & 6 \\ \hline \begin{array}{c}\text { Anti-infectieux (antibiotique, } \\ \text { antifongique, antiparasitaire, } \\ \text { antiviraux) }\end{array} & 32,3 \% & 7 \\ \hline \begin{array}{c}\text { Médicaments du système nerveux } \\ \text { (antidépresseur, neuroleptique, } \\ \text { anxiolytique, antiépileptique, } \\ \text { normothymique) }\end{array} & 14,6 \% & 10 \\ \hline \text { Cytotoxiques } & 11 \\ \hline \text { Immunosuppresseur } & 9,1 \% & 12 \\ \hline \begin{array}{c}\text { Médicaments du système digestif } \\ \text { (antiulcéreux, antiémétique, } \\ \text { antispasmodique, antidiarrhéique, } \\ \text { antilithiasique) }\end{array} & 6,7 \% & 13 \\ \hline \text { Antalgique-Analgésique } \\ \text { (antalgique, AlNS) }\end{array}\right)$


Tableau 3. Définition et illustration des deux types de questions reçues, portant sur la recherche d'interactions médicamenteuses.

Table 3. Definition and examples of the two kinds of questions concerning a search for drug interactions

1. Le patient nécessite la mise en route d'un nouveau traitement. Le médecin demande au pharmacien de vérifier l'absence d'interaction(s). (16/47). Exemples :

- $\quad$ Le patient $X$ est pris en charge en service d'infectiologie. Son traitement habituel comporte Ambisome ${ }^{\circledR}$, Levothyrox ${ }^{\circledR}$, Spasfon $\AA$, Transipeg ${ }^{\circledR}$, Doliprane $\AA$, Vfend $\AA$, Diamox®, Amphotéricine B collyre, Voriconazole collyre, Atropine collyre et Tobradex®. Sur avis de l'ophtalmologiste, le médecin souhaite introduire Diamox §.

- $\quad$ Cas d'une patiente transplantée hépatique chez qui une tuberculose ganglionnaire est suspectée. Elle est actuellement traitée par Cellcept®, Prograf $\AA$, Viread®, Rovalcyte $\AA$. Le prescripteur souhaiterait débuter une quadrithérapie anti-tuberculeuse à base de rifampicine, isoniazide, ethambutol et pyrazinamide.

2. Le prescripteur envisage d'introduire un nouveau traitement avec plusieurs possibilités qu'il communique au pharmacien. A ce dernier revient le rôle d'estimer le rapport bénéfice risque de chacun des scénarios et de se prononcer sur celui qui lui apparaît le plus favorable notamment en termes d'interaction(s). (31/47). Exemple :

- Quelle molécule privilégier (Zyprexa®?, Xeroquel®?, Lithium ?) pour un trouble bipolaire chez une patiente séropositive traitée et en post-transplantation hépatique ? Son traitement habituel associe Xanax $\AA$, Cellcept ${ }^{\circledR}$, Kardegic $\AA$, Ogastoro $\AA$, Calcium vitamine D3®, Prograf $\AA$, Viread $\AA$, Kivexa $\AA$, Prezista ${ }^{\circledR}$ et Norvir $\AA$. 